\title{
ON THE CONNECTION BETWEEN PROPAGATING SOLAR CORONAL DISTURBANCES AND CHROMOSPHERIC FOOTPOINTS
}

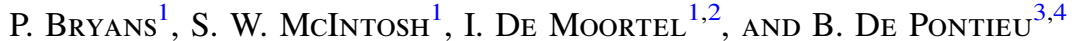 \\ ${ }^{1}$ High Altitude Observatory, National Center for Atmospheric Research, P.O. Box 3000, Boulder, CO 80307, USA \\ ${ }^{2}$ School of Mathematics and Statistics, University of St Andrews, St Andrews, Fife KY16 9SS, UK \\ ${ }^{3}$ Lockheed Martin Solar \& Astrophysics Lab, Org. A021S, Bldg. 252, 3251 Hanover Street, Palo Alto, CA 94304, USA \\ ${ }^{4}$ Institute of Theoretical Astrophysics, University of Oslo, Blindern, P.O. Box 1029 Blindern, NO-0315 Oslo, Norway \\ Received 2016 July 18; revised 2016 September 6; accepted 2016 September 7; published 2016 September 21
}

\begin{abstract}
The Interface Region Imaging Spectrograph (IRIS) provides an unparalleled opportunity to explore the (thermal) interface between the chromosphere, transition region, and the coronal plasma observed by the Atmospheric Imaging Assembly (AIA) of the Solar Dynamics Observatory (SDO). The SDO/AIA observations of coronal loop footpoints show strong recurring upward propagating signals- "propagating coronal disturbances" (PCDs) with apparent speeds of the order of $100-120 \mathrm{~km} \mathrm{~s}^{-1}$. That signal has a clear signature in the slit-jaw images of IRIS in addition to identifiable spectral signatures and diagnostics in the Mg IIh (2803 $⿱$ ) line. In analyzing the Mg IIh line, we are able to observe the presence of magnetoacoustic shock waves that are also present in the vicinity of the coronal loop footpoints. We see there is enough of a correspondence between the shock propagation in $\mathrm{Mg}$ IIh, the evolution of the Si IV line profiles, and the PCD evolution to indicate that these waves are an important ingredient for PCDs. In addition, the strong flows in the jet-like features in the IRIS Si IV slit-jaw images are also associated with PCDs, such that waves and flows both appear to be contributing to the signals observed at the footpoints of PCDs.
\end{abstract}

Key words: Sun: chromosphere - Sun: corona - Sun: magnetic fields - Sun: transition region

Supporting material: animation

\section{INTRODUCTION}

Ever since high-resolution, high-cadence observations of coronal loops became available, propagating intensity perturbations have been observed along large, diffuse coronal loops (Berghmans \& Clette 1999; Schrijver et al. 1999; De Moortel et al. 2000). These quasi-periodic, propagating coronal disturbances (PCDs) are observed to travel at speeds around $100 \mathrm{~km} \mathrm{~s}^{-1}$ with characteristic periods of the order of minutes (typically 3-10 minutes). Based on the propagation speeds, close to the local sound speed, these traveling intensity (density) perturbations were generally interpreted as propagating slow magnetoacoustic waves. We refer the interested reader to De Moortel (2009) for a review of the earlier observations of PCDs.

Various suggestions have been made for the driving mechanism for these coronal PCDs. Waves with similar periods to those of PCDs are ubiquitous in the chromosphere, which has led to the suggestion that slow-mode sound waves propagate from the chromosphere into the corona (De Pontieu et al. 2005). Such chromospheric slow-mode waves have been implicated in driving jet-like features known as dynamic fibrils (De Pontieu et al. 2007a). In particular, these fibrils or Type I spicules have been shown to be driven by slow-mode magnetoacoustic shocks (Hansteen et al. 2006) that exhibit a distinct sawtooth-like signature in chromospheric emission lines (Langangen et al. 2008). More recently, a transition region response to dynamic fibrils has been identified in Interface Region Imaging Spectrograph (IRIS) observations (Skogsrud et al. 2016). However, a clear connection between the shock waves that drive dynamic fibrils and PCDs has not yet been seen in observations.

The work of De Pontieu et al. (2009; in addition to McIntosh $\&$ De Pontieu 2009a, 2009b; De Pontieu et al. 2011) proposed a new picture of how the pieces of the outer solar atmosphere were connected - of how the structures observed could be interpreted as indicators of mass and energy transport into the corona that originate in the magnetized footpoints of the system. Using Solar and Heliospheric Observatory, Hinode, and Solar Dynamics Observatory (SDO) observations, they suggested that the coronal counterpart of rapidly evolving “Type II" chromospheric spicules (De Pontieu et al. 2007b) lay in strongly blueshifted components of asymmetric (UV and EUV) line profiles and the quasi-periodic propagating disturbances that shared common magnetic roots (and velocities; Tian et al. 2011a, 2011b). The observed commonalities between the spicules, the line profile asymmetries, and the PCDs ultimately led to an alternative suggestion for the physical interpretation of the PCDs (De Pontieu \& McIntosh 2010). De Pontieu \& McIntosh (2010) demonstrated that the quasi-periodic nature of the coupled (multi-component) chromospheric-coronal plasma could, in principle, also reproduce the behavior observed in the single-component analysis of spectral data (e.g., Wang et al. 2009) in addition to the PCD amplitudes and speeds inferred from broadband imagers (e.g., De Moortel \& Nakariakov 2012). While Pant et al. (2015) have identified enhanced line widths and intensities in IRIS observations from reconnection-driven quasi-periodic flows at the footpoints of plumes in coronal holes, on the disk, a clear, one-to-one connection between Type II spicules and PCDs has so far not been established.

In addition to the discussion of whether or not these processes can actually sustain the mass requirements of the corona as proposed by De Pontieu et al. (2011), but questioned by Klimchuk (2012), this "waves versus flows" debate has sparked a flurry of activity across the community (e.g., Verwichte et al. 2010; De Moortel et al. 2015). The outcome 
of the debate is in the balance. As a guided slow-mode wave (e.g., De Pontieu et al. 2005), there is no net plasma flow into the corona while, as a flow, they are notoriously difficult to measure with current instrumentation. In both cases, there is difficulty explaining the apparent consistency of the propagation speed (wave or flow) with temperature (Judge et al. 2012; Kiddie et al. 2012) without the magnetic field being heavily involved (e.g., De Pontieu et al. 2011). The resolution to these debates carries some significance. Are Type II spicules and PCDs the manifestation of how the lower and outer solar atmosphere are connected? Are Type II spicules and PCDs the means by which mass is loaded into the (open and closed) heliosphere? There have been suggestions recently from both observations (e.g., Nishizuka \& Hara 2011; Krishna Prasad et al. 2012; Tian et al. 2012; Pant et al. 2015) and numerical modeling (Ofman et al. 2012; Wang et al. 2013) that both interpretations might actually be correct, with waves and flows present on the same structures.

These questions helped drive the requirements of the telescope and spectrograph of IRIS (De Pontieu et al. 2014c). The initial science results of IRIS (e.g., De Pontieu et al. 2014a, 2014b; Peter et al. 2014; Testa et al. 2014; Tian et al. 2014) illustrate the abundance of dynamic (thermal and non-thermal) phenomena occurring at the spatio-temporal resolution limit of the instrument, highlighting the profound complexity of the magneto-thermal structuring of the solar atmosphere.

This Letter presents IRIS observations of the (magnetized) footpoints of coronal loops that display strong multithermal PCDs observed by $S D O /$ Atmospheric Imaging Assembly (AIA). Following a brief discussion of the observations, we analyze the spectral signatures observed at the base of the loop system and trace the chromospheric variability through the transition region and into the corona in the spectral and imaging data.

\section{OBSERVATIONS}

The IRIS spacecraft is in a Sun-synchronous low-Earth orbit and carries a $19 \mathrm{~cm}$ Cassegrain telescope that feeds a dualrange UV spectrograph and slit-jaw imager (SJI), with 0!" 16 pixels and four $2072 \times 1096$ CCDs. The IRIS SJI includes four passbands: two transition region lines (C II $1335 \AA$ and Si IV $1403 \AA$ ), one chromospheric line (Mg II k $2796 \AA$ ), and one photospheric passband (2830 $\mathrm{A}$ ), covering a field of view of $175^{\prime \prime} \times 175^{\prime \prime}$. The IRIS spectrograph has a 0 "! 33 wide and $175^{\prime \prime}$ long slit that covers FUV passbands from $1332 \AA-1358 \AA$ and $1390 \AA$ to $1406 \AA$ and an NUV passband from 2783 to $2834 \AA$.

We analyze two sets of observations in this Letter: 2014 May 15 between 05:26 and 06:44 UT and 2014 September 26 between 00:34 and 01:36 UT. Figure 1 provides IRIS/SJI and $S D O /$ AIA context for the "sit-n-stare" (fixed and tracked slit position on the Sun; IRIS OBSID 3820109554) observations of IRIS.

Co-spatial and co-temporal SDO/AIA observations in the $1600,131,171$, and $193 \AA$ passbands were prepped, (internally) coaligned, and normalized using the SolarSoft a ia_prep routine. These data were then de-rotated to the start time of the IRIS observations and interpolated in time and space to match the IRIS/SJI temporal cadence (9.35 s) and spatial resolution (0!33). Finally, the IRIS and SDO/AIA data sets were coaligned using the first images in the IRIS/SJI (Si IV) $1330 \AA$ and AIA $1600 \AA$ data sets.
As described in Section 1, chromospheric shocks appear as sawtooth-like patterns in the optically thick Mg IIh $(2803 \AA$ ) line. The position of the core reversal of this line swings from blue to red in the presence of a shock. Following the analysis of Leenaarts et al. (2013a, 2013b), we capture the complexity of these line profiles using a double-Gaussian fitting scheme: one Gaussian - the "wing" fit-describes the extended wings of the h-line core while the other-the "core" fit-describes the central reversal. The $\mathrm{Mg}$ IIh profile fit is then the sum of these parts. For the remainder of this work we will concentrate on the centroid of the central reversal, "h3." The position of h3 correlates with the line of sight velocity at optical depth unity (Leenaarts et al. 2013b).

\section{RESULTS}

Figure 2 shows a cutout of the 2014 September 26 observations where we have identified a PCD_corresponding to the solid red lines in panels (C) and (D) of Figure 1. We show three snapshots in the print version and encourage the reader to view the animated version of this figure. The red boxes highlight a Type II spicule in the IRIS/SJI images (top row) and a PCD in the AIA running difference images (second row). The bottom two rows show time-distance plots in running difference along the path of the $\mathrm{PCD} /$ spicule. The red dotted line indicates a propagation speed of $100 \mathrm{~km} \mathrm{~s}^{-1}$. The PCD and the Type II spicule appear to share a common origin, with the PCD extending 5-10 arcsec farther than the spicule. This is consistent with the results of De Pontieu et al. (2011) and Samanta et al. (2015), who studied the connection between spicules and PCDs at the solar limb. Our observations show this clear connection on the disk where there is much less ambiguity than at the limb. In addition, since Type II spicules are associated with flows in chromospheric and TR bandpasses (Rouppe van der Voort et al. 2015), the observed association with PCDs suggests that flows play a role in at least a subset of PCDs.

For the 2014 September 26 observations, the IRIS slit did not coincide with the location of the PCDs and spicules, so there is no spectral information for this event. To explore the spectral signature of spicules and PCDs, we turn to the observations of 2014 May 15. In this case, as shown in panel (A) of Figure 1, the IRIS slit crossed directly over bright fan-like loops where PCDs are present. To further study the connection between $S D O / A I A$ and IRIS data, we have chosen a location on the IRIS slit where the PCDs seen in AIA data appear to originate - a solar Y location of $-376^{\prime \prime}$ and indicated by where the red lines of panels (A) and (B) of Figure 1 intersect the IRIS slit (shown as a dashed red line). Figure 3 shows space-time plots of the SDO/AIA and IRIS/SJI running difference evolution for this location. In this case we use a $62 \mathrm{~s}$ running difference. The long (diagonal) streaks visible in the 193 and $171 \AA$ AIA channels (panels (A) and (B)) are the indicators of PCDs originating at this footpoint. The inclined (blue dashed) reference lines shown in panels (A) and (B) indicate a propagation speed of $120 \mathrm{~km} \mathrm{~s}^{-1}$. The green circles and lines indicate four PCDs that have a strong correlation in the different channels of AIA and in the IRIS SJI. The cooler $131 \AA$ channel (panel (C)) has much poorer signal to noise than those in panels (A) and (B), but we can clearly see the consistent timescales of activity at the bottom of the coronal structure. In the SDO/AIA $304 \AA$ (panel (D)) and IRIS/SJI (panel (E)) space-time plots, we see increasing complexity, with much 

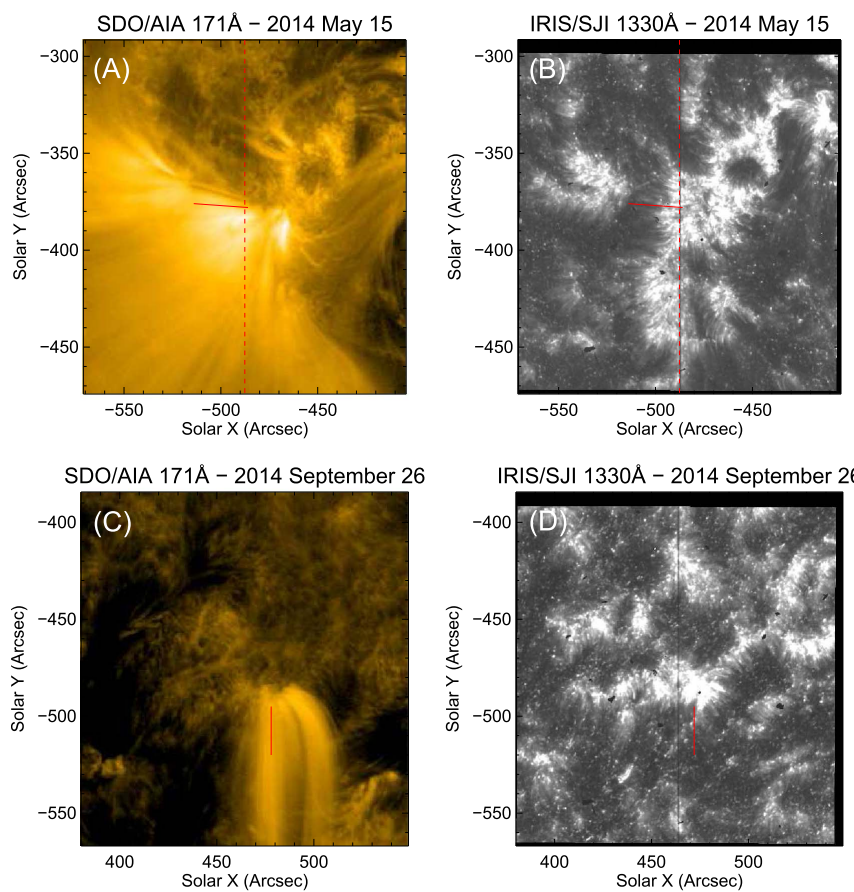

Figure 1. Context images for the PCD observations of 2014 May 15 and 2014 September 26. Panels (B) and (D) show sample $1330 \AA$ slit-jaw images of the "sit-n-stare" time series for the two observations. The red dashed line indicates the position of the IRIS slit in the May observations. Panels (A) and (C) show SDO/AIA $171 \AA$ sub-fields with the same fields of view as the IRIS observations. The solid red curves indicate the locations of the PCDs that we analyze in detail in Section 3.

shorter structures, but again there is a consistency between the patterns and timescales seen in those panels with those of the coronal emission (see also Pereira et al. 2014).

To further explore the signature of PCDs lower in the atmosphere, we turn to the spectral information from IRIS. As outlined in Sections 1 and 2, dynamic fibrils or Type I spicules can be identified by the wavelength of the core reversal of the optically thick Mg IIh line measured by IRIS. In Figure 4, we compare the temporal evolution of the $\mathrm{h} 3$ wavelength with the PCD signature in AIA $171 \AA$. The top panel shows the running difference AIA $171 \AA$ image (same as panel (B) of Figure 3). The red dashed line shows the position of the IRIS slit. The second and third panels of Figure 4 show the spectral evolution of the IRIS data for Si IV $1403 \AA$ and $\mathrm{Mg}$ IIh $2803 \AA$, respectively, for the same $\mathrm{Y}$ position of $-376^{\prime \prime}$. One immediately notices periodic sawtooth-like patterns in the Mg IIh data and corresponding blue shifts in the Si IV data. There also appears to be a correspondence between these features and the PCDs in the AIA running difference image. This connection is best visualized by plotting the parameters shown in the lower panel as a function of time. The black curve is the wavelength of $h 3$ and the red curve is the negative of the AIA $171 \AA$ intensity. The AIA intensity here has been smoothed over the time series and normalized to the h3 wavelength. Plotting the negative AIA intensity helps to compare the timing of the PCDs and the h3 wavelength swing.

The correlation between PCDs in the corona and Type I spicules is evident for the entire time series at the location shown in Figure 4. The h3 wavelength is clearly seen to swing back and forth, symptomatic of the magnetoacoustic shocks that drive dynamic fibrils or Type I spicules. Peaks in the AIA intensity regularly occur at the beginning of these shocks, i.e., where the h3 wavelength swings to the blue. After $\sim 50$ minutes of the observation, the periodicity of the PCDs changes from $\sim 5$ to $\sim 3$ minutes. The magnetoacoustic shocks show a similar change in period at this time, strengthening the argument that these phenomena are related.

\section{DISCUSSION}

The IRIS and SDO observations shown above indicate there is a connection between chromospheric activity and PCDs, but observing that connection is complicated by the geometry and temporal evolution of the interface. De Pontieu et al. (2011) provided a tentative link between chromospheric activity, line profile asymmetries (via Hinode/EIS raster maps), and PCDs. Exploiting the improvements in spectral, spatial, and temporal sampling of the chromosphere and transition region with IRIS over Hinode/EIS, we develop an improved view of the roots of the coronal PCDs.

Demonstrating the connection between chromospheric activity and PCDs is hindered by the difficulty in simultaneously measuring PCDs and their footpoints in the chromosphere. For the 2014 May 15 data set, the IRIS slit crosses a region of fan-like loops where PCDs are evident over several tens of arcseconds. However, most of the observation covers PCDs that have already propagated higher into the corona, away from their footpoints. Given the geometry of this particular active region and the nature of the sit-n-stare operational mode of IRIS, we are only able to identify a single location where PCDs and chromospheric footpoints were observed simultaneously. For this location, however, we can identify a connection between PCDs and Type I spicules.

A further difficulty in connecting chromospheric activity with PCDs in the observation of 2014 May 15 is that PCDs are ubiquitous over the region of coronal loops and happening periodically. Therefore, identifying Type II spicules that correspond with a particular PCD is extremely difficult because of the crowded nature of these events. Using a different observation (2014 September 26), where we can isolate a single PCD, does allow us to connect PCDs to Type II spicules.

Despite the improved resolution and cadence of IRIS, establishing a clear one-to-one correspondence is generally not straightforward due to the effects of line of sight superposition and the geometry of the viewing angle. We have both magnetoacoustic shock (Type I) and jet (Type II) related phenomena, which occur in close vicinity to each other in the observed regions. The complicated nature of establishing a one-to-one connection is illustrated in Figure 5. This figure shows a schematic representation of a chromospheric Type I or Type II spicule (blue), evident in the data as slow magnetoacoustic shocks (Type I), transition region brightenings (orange; Type I), or jets (orange; Type II), and the coronal PCDs (red). The exact location of the IRIS slit will result in either a "hit" where all signatures are captured co-spatially and co-temporally or a "miss," where a one-to-one correspondence will not be clear. Clearly the critical position of the 1D IRIS slit will result in only occasional "hits" and a lot of "misses." To obtain a larger statistical sample of one-to-one connections between PCDs, and the chromospheric or TR counterparts will require an improved observation sequence where, rather than IRIS being in sit-n-stare mode, the slit rasters rapidly across a region of PCDs. 

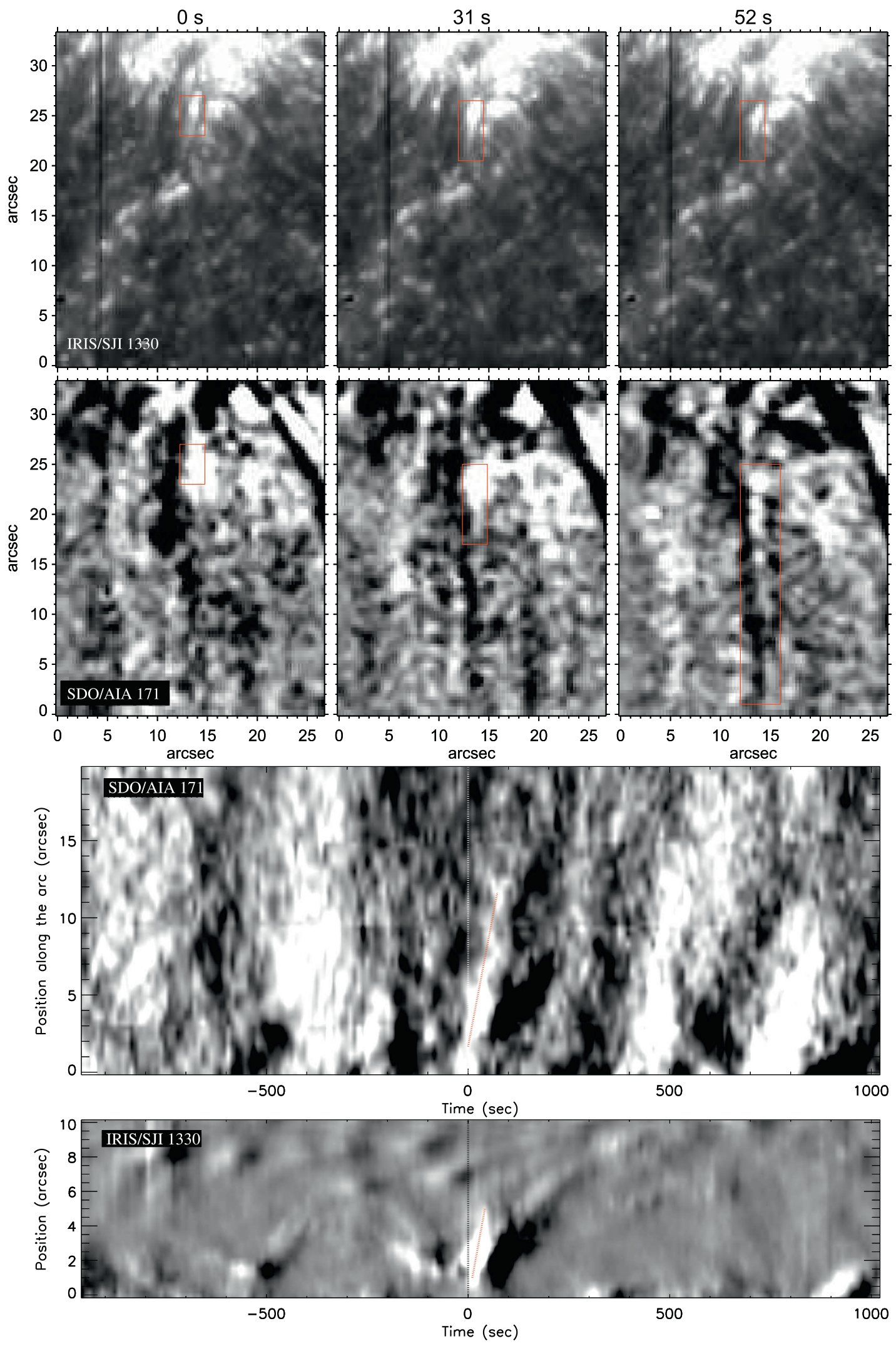

Figure 2. Upper six panels show snapshots from an animation of a Type II spicule and PCD from the 2014 September 26 data set. The top row shows the IRIS/SJI images, and the second row shows the SDO/AIA 171 running difference images. The red boxes highlight the location of the spicule and PCD. The lower two panels show time-distance plots of the same region in running difference for both AIA 171 (third row) and IRIS/SJI (bottom row). The red dotted line corresponds to a propagation speed of $\sim 100 \mathrm{~km} \mathrm{~s}^{-1}$.

(An animation of this figure is available.) 


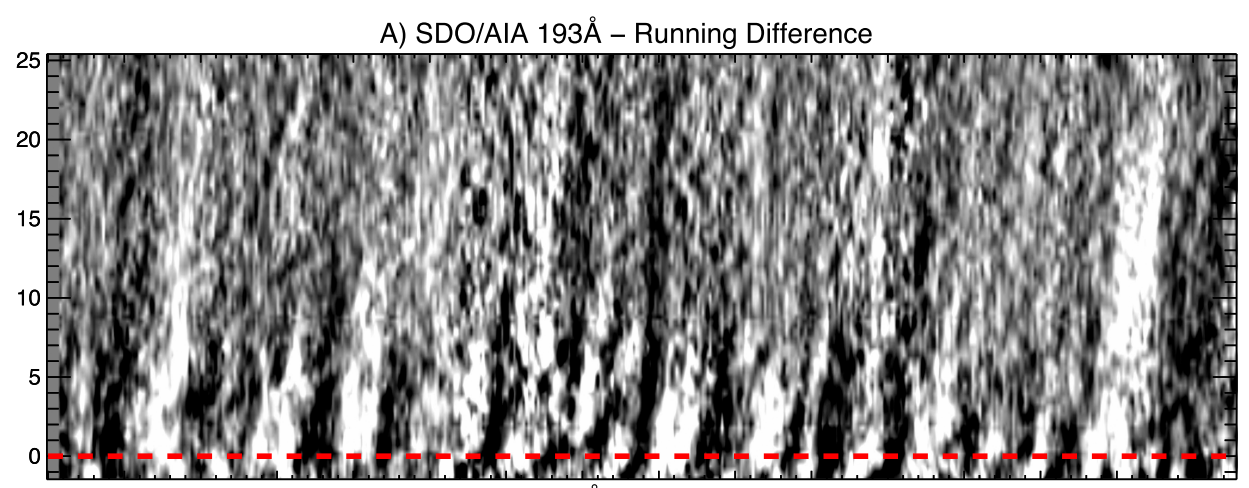

B) SDO/AIA $171 \AA ̊$ - Running Difference
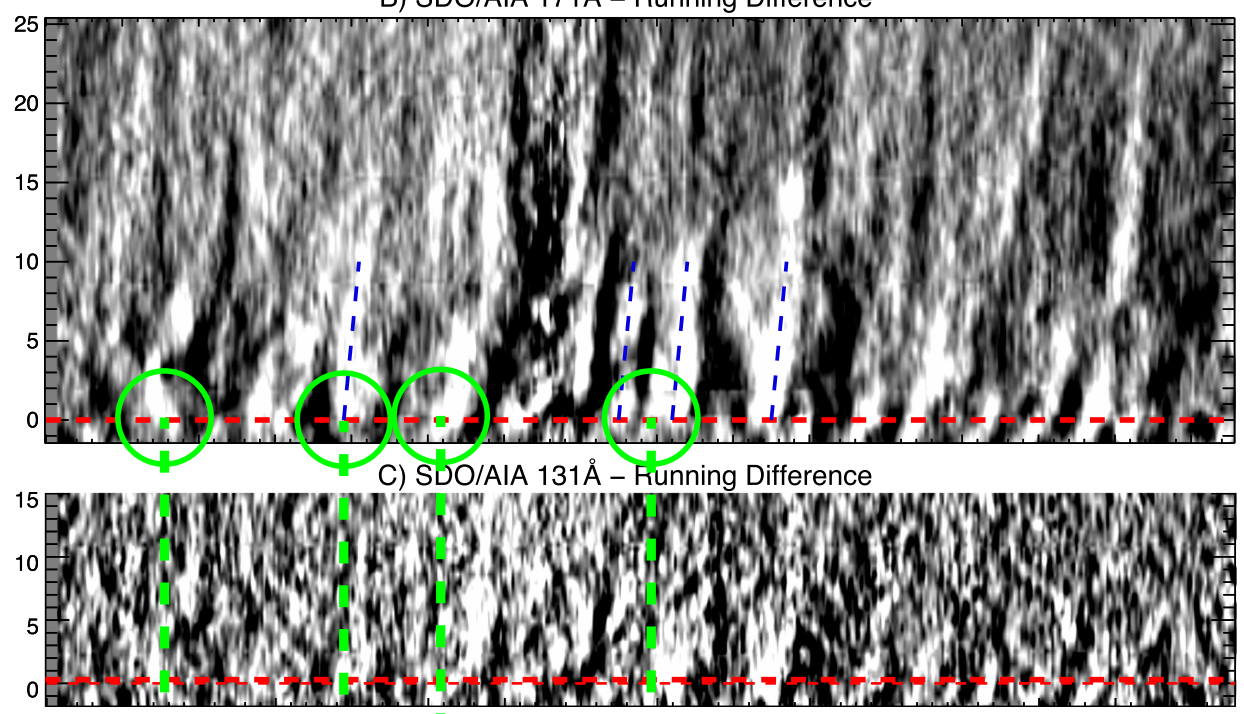

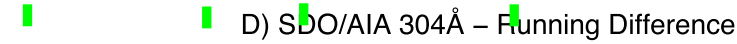
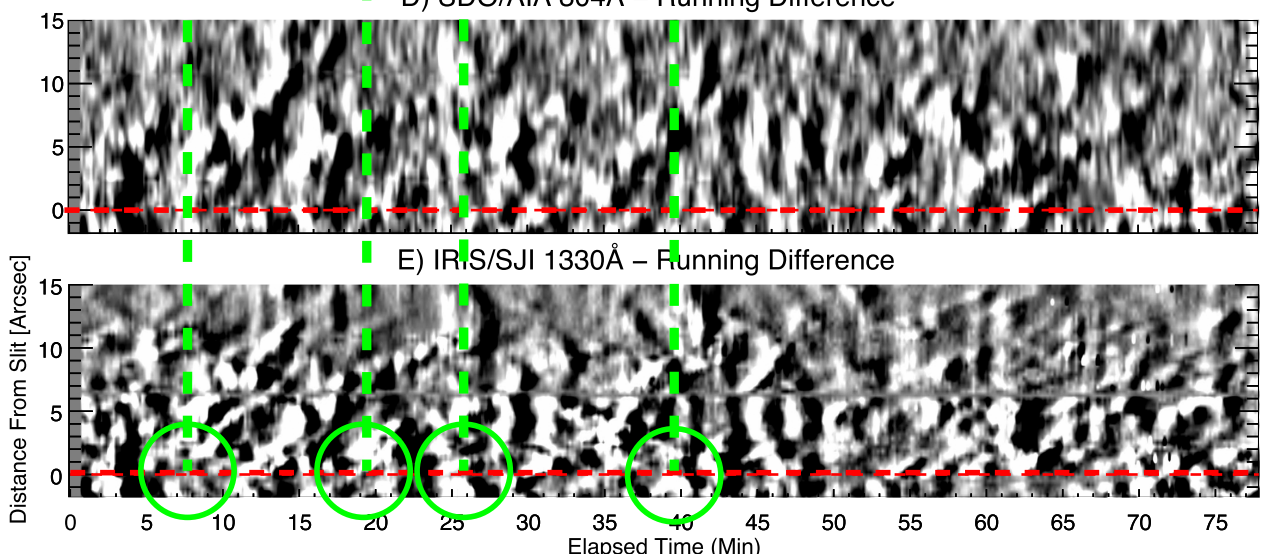

Figure 3. From bottom to top we show the thermal variation of the space-time plots at $y=-356^{\prime \prime}$ for SDO/AIA $193 \AA$ (A), $171 \AA$ (B), $131 \AA$ (C), $304 \AA$ (D), and IRIS SJI $1330 \AA$ (E) for the 2014 May 15 data set. In each case, the location of the IRIS slit is shown as a thick red dashed line. For reference, panel (B) shows inclined blue dashed lines that indicate an apparent speed of $120 \mathrm{~km} \mathrm{~s}^{-1}$ of the propagating coronal disturbances observed. The green circles and lines are drawn to indicate particularly strong correspondences in the observational sequence.

In addition to the limitations of the $1 \mathrm{D}$ slit, the geometry and/or magnetic flux of the footpoints appear to be changing very rapidly - on timescales comparable to the periods of the PCDs-further complicating the one-to-one correspondence between chromospheric dynamics and the coronal PCDs. This is a problem that becomes further compounded by pushing to higher and higher spatial resolution measurements-emphasizing the need to acquire two-dimensional spectral information about the interface.

\section{CONCLUSION}

This Letter uses IRIS data to identify the chromospheric and transition region dynamics associated with PCDs observed by AIA. Despite the complexity of connecting chromospheric, TR, and coronal dynamics, our results show that the wavelength coverage of IRIS and the improved spatial and temporal resolution allow a one-to-one connection, but only when the viewing geometry allows. In particular, we find nice examples where both shock waves (Type I spicules or dynamic fibrils) 


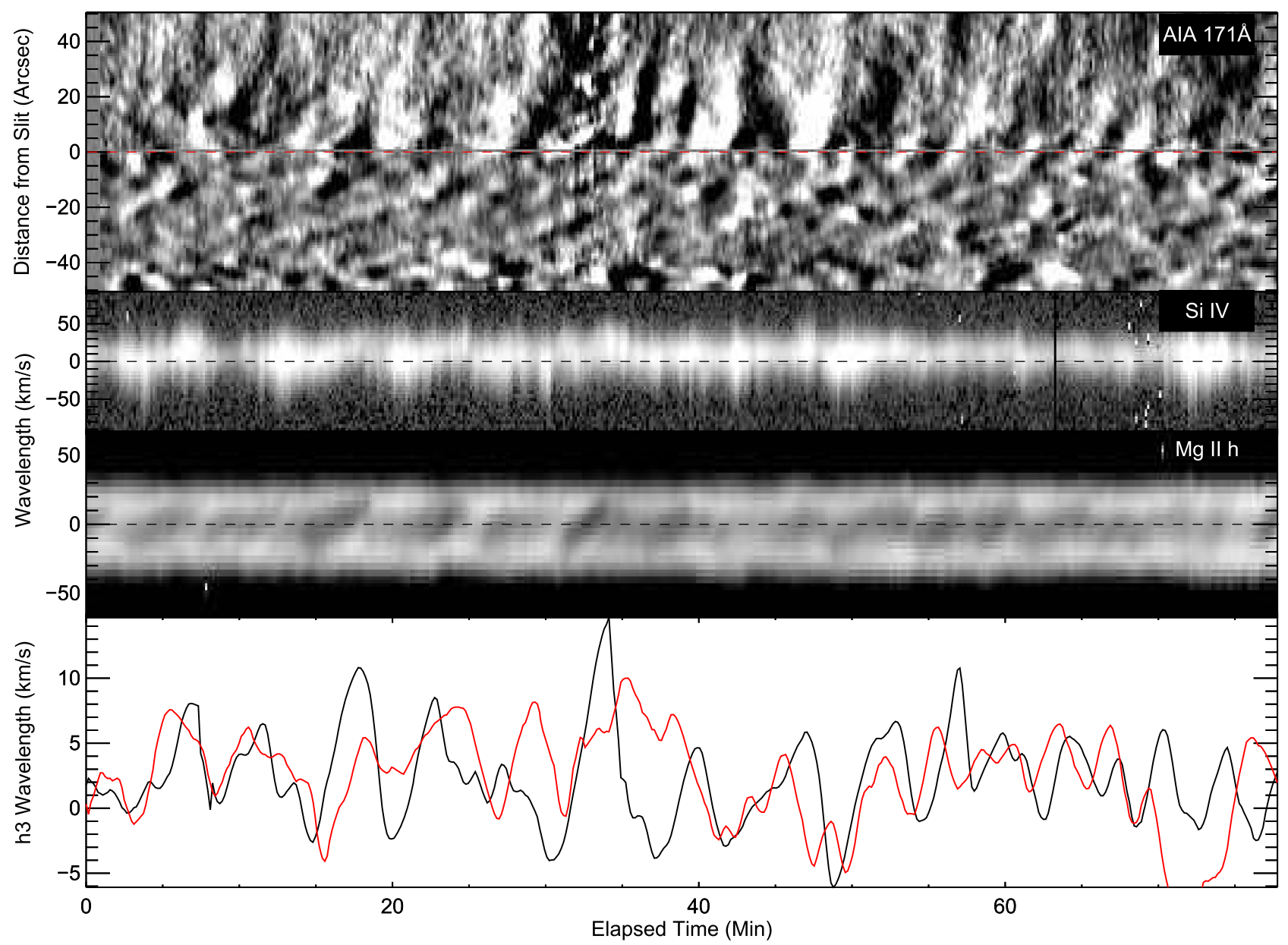

Figure 4. Top three rows show (from top to bottom) a running time difference of SDO/AIA $171 \AA$ and " $\lambda$-time" plots of Si IV (1403 $\AA$ ) and Mg IIh (2803 $\AA$; 2014 May 15). The location of the IRIS slit in the running difference is shown as a red dashed line, while the horizontal (black) dashed lines in the other three panels show the zero wavelength positions. The lowest panel shows the $\mathrm{h} 3$ wavelength (black line) and the negative of the AIA 171 A intensity (red line). The AIA intensity has been normalized to the $\mathrm{h} 3$ wavelength for ease of comparison.

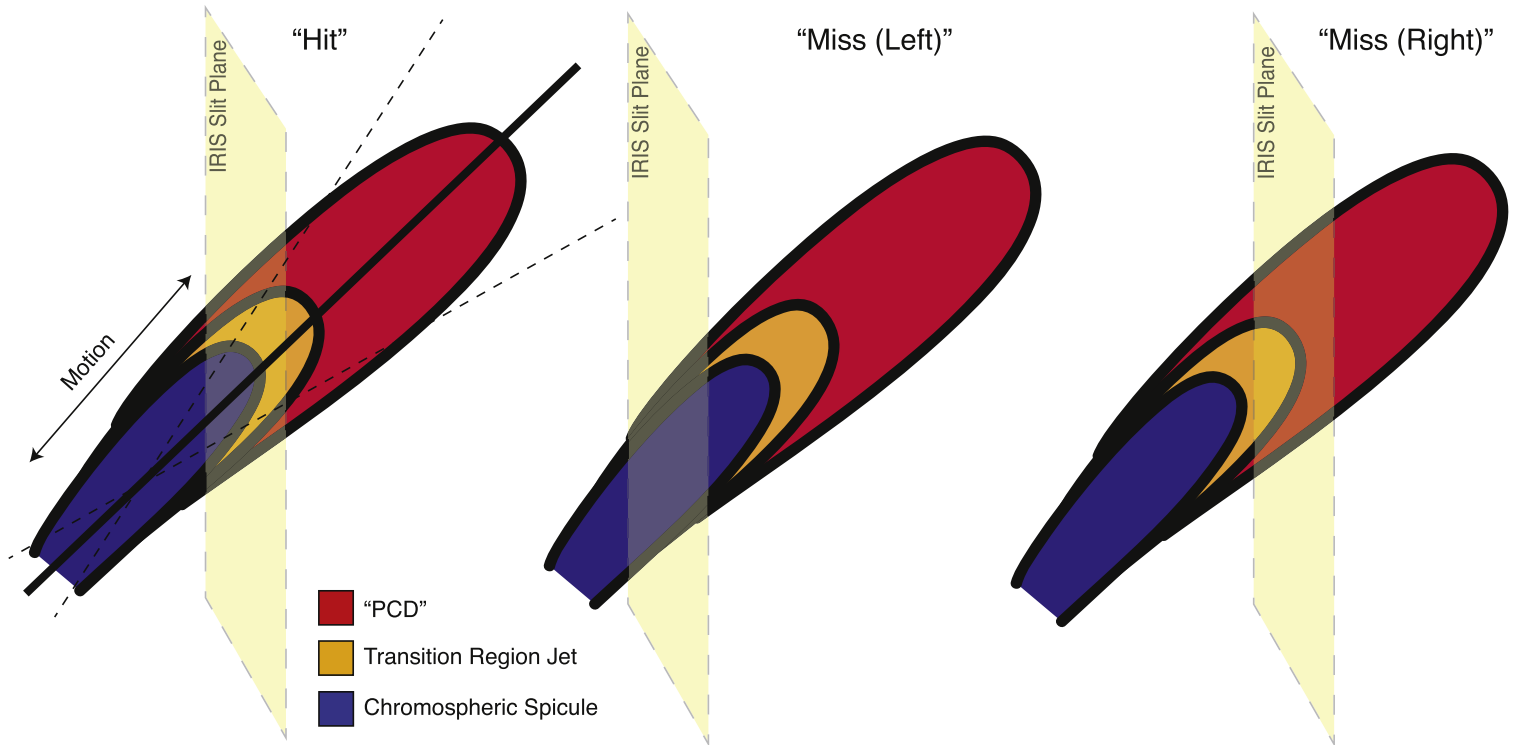

Figure 5. Illustration of the impact that geometry can have on establishing the chromo-coronal connection via the chromospheric spicule (Type I and or Type II; blue), the corresponding transition region brightening or jet (orange) and coronal PCD (red). For Type I spicules, the TR counterpart is located at the top of the chromospheric jet and appears as a short-lived, round brightening (Skogsrud et al. 2016). For Type II spicules, the TR counterpart is a linear, jet-like feature, which forms during the spicule's lifetime and plasma is heated all along the chromospheric Type II spicule (Pereira et al. 2014; Skogsrud et al. 2016). From left to right we show the "hit" and "miss" scenarios discussed in the body of the text. 
and strong flows (Type II spicules) are connected with PCDs, illustrating that both waves and flows appear to be involved at the footpoints of PCDs.

IRIS is a NASA Small Explorer developed and operated by LMSAL with mission operations executed at NASA Ames Research center and major contributions to downlink communications funded by ESA and the Norwegian Space Center. This work is supported by NASA contract NNG09FA40C (IRIS) and has benefited from discussions at the International Space Science Institute (ISSI) meeting on "Heating of the Magnetized Chromosphere." The visit of S.M.C. to St Andrews was partly supported by a Royal Society of Edinburgh Research Visitor to Scotland Grant. The research leading to these results has also received funding from the UK Science and Technology Facilities Council, the European Commission Seventh Framework Programme (FP7/2007-2013) under the grant agreement SOLSPANET (project No. 269299), and the European Union Horizon 2020 research and innovation programme (grant agreement No. 647214).

\section{REFERENCES}

Berghmans, D., \& Clette, F. 1999, SoPh, 186, 207

De Moortel, I. 2009, SSRv, 149, 65

De Moortel, I., Antolin, P., \& Van Doorsselaere, T. 2015, SoPh, 290, 399

De Moortel, I., Ireland, J., \& Walsh, R. W. 2000, A\&A, 355, L23

De Moortel, I., \& Nakariakov, V. M. 2012, RSPTA, 370, 3193

De Pontieu, B., Erdélyi, R., \& De Moortel, I. 2005, ApJL, 624, L61

De Pontieu, B., Hansteen, V. H., Rouppe van der Voort, L., van Noort, M., \& Carlsson, M. 2007a, ApJ, 655, 624

De Pontieu, B., \& McIntosh, S. W. 2010, ApJ, 722, 1013

De Pontieu, B., McIntosh, S. W., Carlsson, M., et al. 2011, Sci, 331, 55

De Pontieu, B., McIntosh, S. W., Hansteen, V. H., et al. 2007b, PASJ, 59, S655
De Pontieu, B., McIntosh, S. W., Hansteen, V. H., \& Schrijver, C. J. 2009, ApJL, 701, L1

De Pontieu, B., Rouppe van der Voort, L., McIntosh, S. W., et al. 2014b, Sci, 346, 1255732

De Pontieu, B., Title, A., \& Carlsson, M. 2014a, Sci, 346, 315

De Pontieu, B., Title, A. M., Lemen, J. R., et al. 2014c, SoPh, 289, 2733

Hansteen, V. H., De Pontieu, B., Rouppe van der Voort, L., van Noort, M., \& Carlsson, M. 2006, ApJL, 647, L73

Judge, P. G., de Pontieu, B., McIntosh, S. W., \& Olluri, K. 2012, ApJ, 746, 158

Kiddie, G., De Moortel, I., Del Zanna, G., McIntosh, S. W., \& Whittaker, I. 2012, SoPh, 279, 427

Klimchuk, J. A. 2012, JGRA, 117, A12102

Krishna Prasad, S., Banerjee, D., \& Singh, J. 2012, SoPh, 281, 67

Langangen, Ø., Carlsson, M., Rouppe van der Voort, L., Hansteen, V., \& De Pontieu, B. 2008, ApJ, 673, 1194

Leenaarts, J., Pereira, T. M. D., Carlsson, M., Uitenbroek, H., \& De Pontieu, B. 2013a, ApJ, 772, 89

Leenaarts, J., Pereira, T. M. D., Carlsson, M., Uitenbroek, H., \& De Pontieu, B. 2013b, ApJ, 772, 90

McIntosh, S. W., \& De Pontieu, B. 2009a, ApJ, 707, 524

McIntosh, S. W., \& De Pontieu, B. 2009b, ApJL, 706, L80

Nishizuka, N., \& Hara, H. 2011, ApJL, 737, L43

Ofman, L., Wang, T. J., \& Davila, J. M. 2012, ApJ, 754, 111

Pant, V., Dolla, L., Mazumder, R., et al. 2015, ApJ, 807, 71

Pereira, T. M. D., De Pontieu, B., Carlsson, M., et al. 2014, ApJL, 792, L15

Peter, H., Tian, H., Curdt, W., et al. 2014, Sci, 346, 1255726

Rouppe van der Voort, L., De Pontieu, B., Pereira, T. M. D., Carlsson, M., \& Hansteen, V. 2015, ApJL, 799, L3

Samanta, T., Pant, V., \& Banerjee, D. 2015, ApJL, 815, L16

Schrijver, C. J., Title, A. M., Berger, T. E., et al. 1999, SoPh, 187, 261

Skogsrud, H., Rouppe van der Voort, L., \& De Pontieu, B. 2016, ApJ, 817, 124

Testa, P., De Pontieu, B., Allred, J., et al. 2014, Sci, 346, 1255724

Tian, H., DeLuca, E. E., Cranmer, S. R., et al. 2014, Sci, 346, 1255711

Tian, H., McIntosh, S. W., De Pontieu, B., et al. 2011a, ApJ, 738, 18

Tian, H., McIntosh, S. W., Habbal, S. R., \& He, J. 2011b, ApJ, 736, 130

Tian, H., McIntosh, S. W., Wang, T., et al. 2012, ApJ, 759, 144

Verwichte, E., Marsh, M., Foullon, C., et al. 2010, ApJL, 724, L194

Wang, T., Ofman, L., \& Davila, J. M. 2013, ApJL, 775, L23

Wang, T. J., Ofman, L., Davila, J. M., \& Mariska, J. T. 2009, A\&A, 503, L25 